\title{
UV exposure and melanoma prognostic factors: results from Clinical National Melanoma Registry (CNMR)
}

\author{
Maurizio Montella ${ }^{1 *}$, Sara Gandini ${ }^{2}$, Carlo Riccardo Rossi ${ }^{3}$, Alessandro Testori ${ }^{2}$, Giuseppe Mastrangelo ${ }^{3}$, \\ Diego Serraino ${ }^{4}$, Mario Budroni ${ }^{5}$, Anna Crispo ${ }^{1}$, Antonio Maria Grimaldi ${ }^{2}$, Paolo Antonio Ascierto ${ }^{1}$, CNMR GROUP ${ }^{1}$
}

From Melanoma Bridge meeting 2013

Naples, Italy. 5-8 December 2013

\section{Background}

Previous studies have reported an association between sun exposure and improved cutaneous melanoma (CM) survival. We analysed the association of UV exposure with prognostic factors and outcome in the National Clinical Melanoma Registry.

\section{Methods}

Clinical and socio-demographic features were collected melanoma patients at diagnosis, together with information on sunbed exposure and sunny holidays. Analyses were carried out to investigate the associations between UV exposure and melanoma prognostic factors.

\section{Results}

From December 2010, we retrieved information from 3299 melanoma patients from 39 IMI (Intergruppo Melanoma Italiano) sites geographically representative. $40 \%$ of the patients are over 60 years old, $52 \%$ are men, $49 \%$ live in the north of Italy and 56\% are at high educational level (at least high school). $8 \%$ of the patients have melanoma familiarity and $55 \%$ have fair phenotype (Fitzpatrick skintype I or II). $2 \%$ have metastases and $11 \%$ lymph-node involvement. $8 \%$ have very thick melanoma (Breslow $>4$ $\mathrm{mm}$ ) and $21 \%$ ulcerated melanoma.

Holidays in the sun five years before CM diagnosis, among non-metastatic patients, were significantly associated with lower Breslow thickness $(\mathrm{p}=0.003)$, after multiple adjustment including socio-demographic status, degree of doctor making the diagnosis and residence. Sunbed exposure is not significantly associated with Breslow thickness.

\section{Conclusions}

Holidays in the sun were associated with thinner melanomas. However, these results do not prove a direct causal effect of sun exposure on survival since other confounding factors, such as Vitamin D serum levels, may play a role.

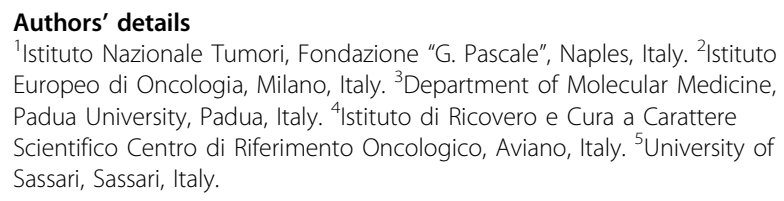
Europeo di Oncologia, Milano, Italy. ${ }^{3}$ Department of Molecular Medicine, Padua University, Padua, Italy. ${ }^{4}$ Istituto di Ricovero e Cura a Carattere Scientifico Centro di Riferimento Oncologico, Aviano, Italy. ${ }^{5}$ University of Sassari, Sassari, Italy.

Published: 6 May 2014

\section{References}

1. Berwick M, Armstrong BK, Ben-Porat L, Fine J, Kricker A, et al: Sun exposure and mortality from melanoma. J Natl Cancer Inst 2005, 97:195-199.

2. Rosso S, Sera F, Segnan N, Zanetti R: Sun exposure prior to diagnosis is associated with improved survival in melanoma patients: Results from a long-term follow-up study of Italian patients. Eur J Cancer 2008, 44:1275-1281.

3. Yang L, Lof M, Veierod MB, Sandin S, Adami HO, et al: Ultraviolet exposure and mortality among women in sweden. Cancer Epidemiol Biomarkers Prev 2011, 20:683-690.

4. Gandini Sara, De Vries Esther, Tosti Giulio, Botteri Edoardo, Spadola Giuseppe, et al: Sunny holidays before and after melanoma diagnosis are respectively associated with lower Breslow thickness and lower relapse rates in Italy. Plos One.

\section{doi:10.1186/1479-5876-12-S1-P3}

Cite this article as: Montella et al.: UV exposure and melanoma prognostic factors: results from Clinical National Melanoma Registry (CNMR). Journal of Translational Medicine 2014 12(Suppl 1):P3.

\footnotetext{
* Correspondence: m.montella@istitutotumori.na.it

${ }^{1}$ Istituto Nazionale Tumori, Fondazione "G. Pascale", Naples, Italy

Full list of author information is available at the end of the article
} 